\title{
Paysages en mouvement : pour une esthétique de la transformation
}

\author{
Landscapes in motion: for an aesthetic of transformation
}

\author{
Gianni Burattoni ${ }^{1}$ \\ ${ }^{1}$ artiste-paysagiste
}

RÉSUMÉ. Gianni Burattoni a participé, en tant qu'artiste spécialiste du jardin et du paysage, à un projet de réhabilitation paysagère à grande échelle, dans la région de la Goitsche en Allemagne. II s'agissait de reconvertir cette ancienne région industrielle en un "paysage culturel ", associant des enjeux culturels, écologiques et esthétiques. Cet article présente les choix artistiques ayant guidé l'aménagement du "Carré Vert », avec des installations artistiques conçues par l'auteur. Ces choix étaient fondés sur la reconnaissance des transformations perpétuelles des paysages, au-delà des distinctions entre l'évolution naturelle et l'activité humaine. Ils prennent en compte l'histoire industrielle des lieux, ainsi que leurs caractéristiques écologiques actuelles, pour proposer une nouvelle manière de les habiter.

ABSTRACT. Gianni Burattoni participated, as an artist specialist of garden and landscape, in a large-scale landscape rehabilitation project in the Goitsche region of Germany. The idea was to convert this former industrial region into a "cultural landscape", combining cultural, ecological and aesthetic issues. This article presents the artistic choices that guided the development of the "Green Square", including artistic installations designed by the author. These choices were based on the recognition of the perpetual transformations of landscapes, beyond the distinctions between natural evolution and human activity. They take into account the industrial history of the places, as well as their current ecological characteristics, to propose a new way of living them.

MOTS-CLÉS. art, paysage, aménagement, installation artistique, réhabilitation, paysage industriel.

KEYWORDS. art, landscape, landscaping, art installation, rehabilitation, industrial landscape.

Ce titre a été emprunté au livre de Massimo Venturi-Ferriolo, philosophe italien du jardin et du paysage, édité en $2016 \mathrm{chez}$ Derive e Approdi. En voici quelques extraits significatifs et emblématiques en guise d'introduction pour le sujet que nous allons aborder.

«(...) Un refrain perpétuel et immuable, nourri par le besoin de certitudes, revient quasiment régulièrement : «Qu'est-ce que le paysage?»

Le mot fait partie du langage quotidien ainsi que de celui des médias. Il traverse de nombreux domaines disciplinaires dans nos académies et universités avec une référence constante au milieu dit «naturel», au panorama, à l'œuvre d'art patrimoniale qu'il faut sauvegarder, au lieu vécu. Tant de voix contradictoires caractérisent son utilisation. L'article défini - le - abstrait souvent un paysage de la réalité qui lui est propre et perceptible à travers son essence narrative, donc lisible dans les lieux de vie pour en apercevoir la source et le processus. (...)

(...) Le nom et la chose appartiennent à une réalité en mouvement, simple et complexe, insérée dans l'incessant mouvement dont parle Héraclite: rien ne reste immuable, tout se transforme. Les lieux perdent au fur et à mesure leur lisibilité. Nous n'arrivons pas à gouverner nos paysages qui sont désormais homologués par le marché et par la globalisation. La spéculation immobilière et financière les dégrade. (...) 
(...) Une question nous inquiète : avons-nous perdu le paysage ? Elle est formulée avec l'article défini, comme si on parlait d'une entité unique, du monde entier vu par satellite, abstrait, comme La Plaine de la Vérité de Platon, ou l’Éden. (...)

(...) Les lieux ne meurent pas. Ils se modifient parce qu'ils subissent une crise, une transition d'un état à un autre. Nous ne pouvons donc pas parler de « mort du paysage », mais plutôt de nouveaux lieux, de transformation rapide, incessante, incontrôlée. (...)

(...) Un monde visible, constitué par la totalité culturelle, éthique et esthétique des actions humaines, que nous pouvons dominer panoramiquement des yeux, s'ouvre au regard. Les hommes l'ont créé et ils l'habitent en le transformant sans cesse pour exister : un continuel habiter et construire. Lieu commun d'un ensemble de lieux, chaque paysage est un ethos où chaque habitant a son propre rôle et détient une mesure : un paysage qui embrasse la totalité de la vie avec ses formes visibles et invisibles, matérielles et immatérielles, et avec l'esprit et ses symboles.

L'action humaine agit en modifiant l'environnement. L'esthétique réfléchit sur les résultats de celle-ci : elle ne considère pas seulement la forme, elle est éthique visible concrètement dans l'espace, elle est une manifestation de liberté, d'un choix, d'une volonté de goût. L'éthique est la vie active avec ses relations entre individus, entre moi et les autres, comme vice-versa les autres entre eux : un tissu en continuel mouvement qui produit un monde esthétique, en lui donnant une justification. (...)

(...) L'être humain, actif par son essence même, incapable de vivre dans des sites vierges et originels, remplace avec l'intelligence ses propres déficiences organiques par la création d'un monde utile à son existence. Il transforme, par le travail, la nature d'un lieu en un réceptacle complexe mais fonctionnel. En soignant son monde, il crée sa propre culture.

Activité sédimentée dans l'histoire et résultat du projet en tant que modification visible, ce que nous considérons culture comprend le patrimoine matériel et spirituel de chaque société, en laissant une empreinte symbolique dans le territoire où elle agit. Sa valeur éthique caractérise un paysage avec ses produits manufacturés évidents et avec un patrimoine intellectuel diffus et hétérogène, parfois enserré harmonieusement, parfois en antagonisme avec la réalité visible et la tradition cachée. Tous les éléments de la totalité d'une culture y participent, entrelacés et mêlés, et ils tissent une trame composée de mythes, traditions, valeurs, normes, langages, symboles, habitudes, comportements, mentalités, institutions, systèmes économiques et sociétés. Ce sont tous des points d'une relation, des expressions éthiques d'un lieu, ou accueillis par celui-ci à travers la transformation multiculturelle et inter-ethnique. (...)»

La modification des choses est liée à l'existence même. Tout processus de paysage a comme base un projet qui ouvre sur l'avenir. Le projet dont nous allons parler n'élabore pas seulement des objets, mais des relations. Le projet est un désir qu'il faut transformer en réalité, il est l'anticipation mentale et/ou dessinée de la possibilité d'un lieu et d'un monde autre.

La transformation de la région de la Goitsche en Allemagne, dans un laps de temps très court, est emblématique de la pérennité des lieux, même s’ils ont été modifiés.

Le projet du «Carré vert » (un parc péri-urbain sur un carreau de mine) dans le territoire de Bitterfeld en ex-RDA est emblématique des préoccupations écologiques actuelles et des exigences de pluridisciplinarité que tout projet de paysage doit porter. 
Ce projet est le fruit d'un travail d'équipe impulsé et mené pendant quatre ans par Jacques Leenhardt, directeur du projet et directeur d'études à l'École des hautes études en sciences sociales. Les autres membres de l'équipe : Heike Brückner, paysagiste de la Fondation Bauhaus à Dessau, Gianni Burattoni, artiste paysagiste italien, Siegfried Knoll, paysagiste, Ökoplan GmbH Leipzig, Bernd Neugebauer, ingénieur forestier de Trees for people GmbH à Freiburg et Thomas Worms, ingénieur en hydraulique, collaborateur aux réalisations et membre de 1'Expo 2000 à Dessau.

En tant que membre de l'équipe, je laisse parler Jacques Leenhardt, avec son texte clair et synthétique, paru dans Les Annales de la recherche urbaine $\mathrm{n}^{\circ}$ 85, en 1999, sous le titre : «Le Carré vert, un parc périurbain sur un carreau de mine ».

« Il était une fois une Europe dynamique dont la richesse était fondée sur les industries du fer, du charbon et de la chimie. L'Allemagne, l'Angleterre, la Belgique, la France, la Tchécoslovaquie et la Pologne se sont développées, tout au long de la révolution industrielle, selon un modèle répondant aux exigences de ces industries. Dans cette histoire, qui s'est close avec le XXe siècle, la région Sachsen-Anhalt a joué un rôle particulièrement illustre, et le complexe chimique de Bitterfeld-Wolfen reste dans la mémoire de cette épopée industrielle un des hauts-lieux les plus représentatifs.

Mais les temps ont changé. Les sources de profit et de bien-être sont aujourd'hui évaluées différemment d'il y a quelques décennies. Les sociétés ont également pris conscience du fait que la pollution des sols et de l'air est une menace pour les générations à venir. Les industries tertiaires offrent de nouvelles chances de développement mais en même temps exigent des comportements nouveaux et une attention sourcilleuse à la qualité de l'environnement.

Dans ce nouveau contexte qui affecte la Région Sachsen-Anhalt comme l'ensemble des pays européens, de nouvelles orientations ont été données aux projets de développement. C'est ainsi qu'est né, sous l'impulsion de 1'EXPO 2000 qui aura lieu à Hanovre, le programme «Kulturlandschaft Goitzsche» (Paysage de culture dans la Goitzsche) au sein de l'ensemble qui a reçu le nom de « Triangle de la Réforme ».

Cette appellation se réfère à l'existence de trois pôles dont la puissance culturelle, politique et économique a donné sa force à cette région au cours des derniers siècles. Elle relie trois foyers d'invention et de modernité symbolisés par trois villes ayant fourni une contribution originale au développement de l'Allemagne: Wittenberg, berceau de la Réforme luthérienne, par laquelle aussi la langue allemande allait se voir, pour la première fois, proprement établie dans sa structure ; Bitterfeld dont le nom restera lié aux inventions les plus prometteuses de la chimie, notamment celle de la photographie en couleur, et enfin Dessau, où l'installation du Bauhaus par Walter Gropius en 1926 marque une révolution à la fois dans l'architecture, la pédagogie et les relations entre culture et industrie.

Le Projet « Kulturlandschaft Goitzsche » vise à déterminer des axes de développement pour le territoire de l'ancienne mine de lignite à ciel ouvert de la Goitzsche, et par conséquent pour la ville de Bitterfeld et ses environs. Il accompagne les mesures de restructuration qui doivent réactiver le tissu industriel de la Région, notamment autour des nouvelles technologies. Il indique aussi la volonté d'aménager le territoire dévasté de manière à lui donner une nouvelle vocation touristique, impliquant la création d'équipements de loisirs. 
L'image très négative qui pèse sur la ville de Bitterfeld, du fait de l'extraordinaire pollution chimique provoquée par l'industrie, ne pourra être transformée qu'en offrant un projet exemplaire dans lequel les préoccupations écologiques et esthétiques prendront une place déterminante.

Le projet que nous présentons pour le Goitzsche-Wald s'inscrit également dans l'histoire ouverte par le Prince Franz von Sachsen-Dessau, qui a légué à cette Région un des plus beaux ensembles de jardins de conception romantique: le Gartenreich. Il s'appuie donc sur une histoire culturelle, industrielle et écologique riche et contrastée. »

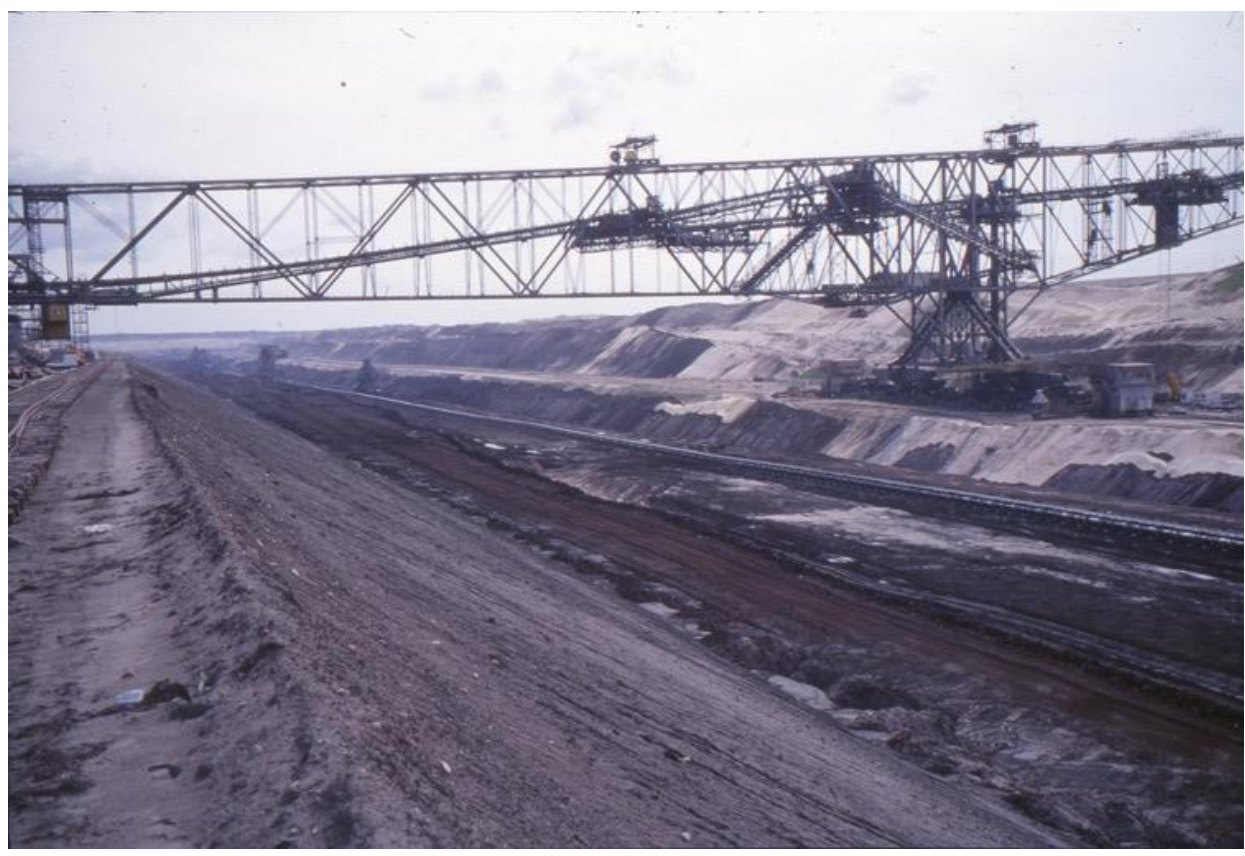

Illustration 1 : Paysage de la Goitzsche, ravagé par l'industrie minière

\section{Autour de la ville de Bitterfeld}

Le projet «Kulturlandschaft Goitzsche » a déterminé quatre territoires dans les cent kilomètres carrés qui constituent la Goitzsche, lesquels devront, chacun sur un mode différent, offrir une variété de paysages et d'activités liées à la culture et à la nature, permettant d'intéresser des publics larges et variés.

\section{Le port et la promenade}

La ville de Bitterfeld s'ouvre aujourd'hui sur les eaux d'un lac qui vient remplir l'immense trou laissé par la mine. Un concours d'idées gagné par le Français Gilles Vexlard et le bureau Latitude Nord a avalisé la construction d'une large promenade en bord de lac permettant à la ville de se retourner vers l'espace auquel elle tournait le dos.

\section{L'Agora et la zone d'activités culturelles}

Une vaste presqu'île accueillera des équipements culturels conformément à un projet du paysagiste allemand Siegfried Knoll ainsi que l'œuvre de plusieurs artistes sélectionnés par le Conseil d'orientation du projet. 


\section{Les espaces d'intersection}

Étant donné que la mine est venue briser le cours ancien des rivières et les voies de circulation reliant plusieurs villages effacés de la carte, un projet a été développé par l'architecte italien Domenico Lucciani pour restituer dans un nouveau paysage quelques éléments de cette mémoire topographique.

\section{Un parc dans la Goitzsche-Wald}

La partie la plus ancienne de la mine a été replantée dans les années soixante. Elle porte le nom de Goitzsche-Wald, la forêt de la Goitzsche. C'est sur ce territoire de 2000 hectares que j'ai développé, avec une équipe pluridisciplinaire, le projet auquel nous avons donné le nom : Le Carré Vert.

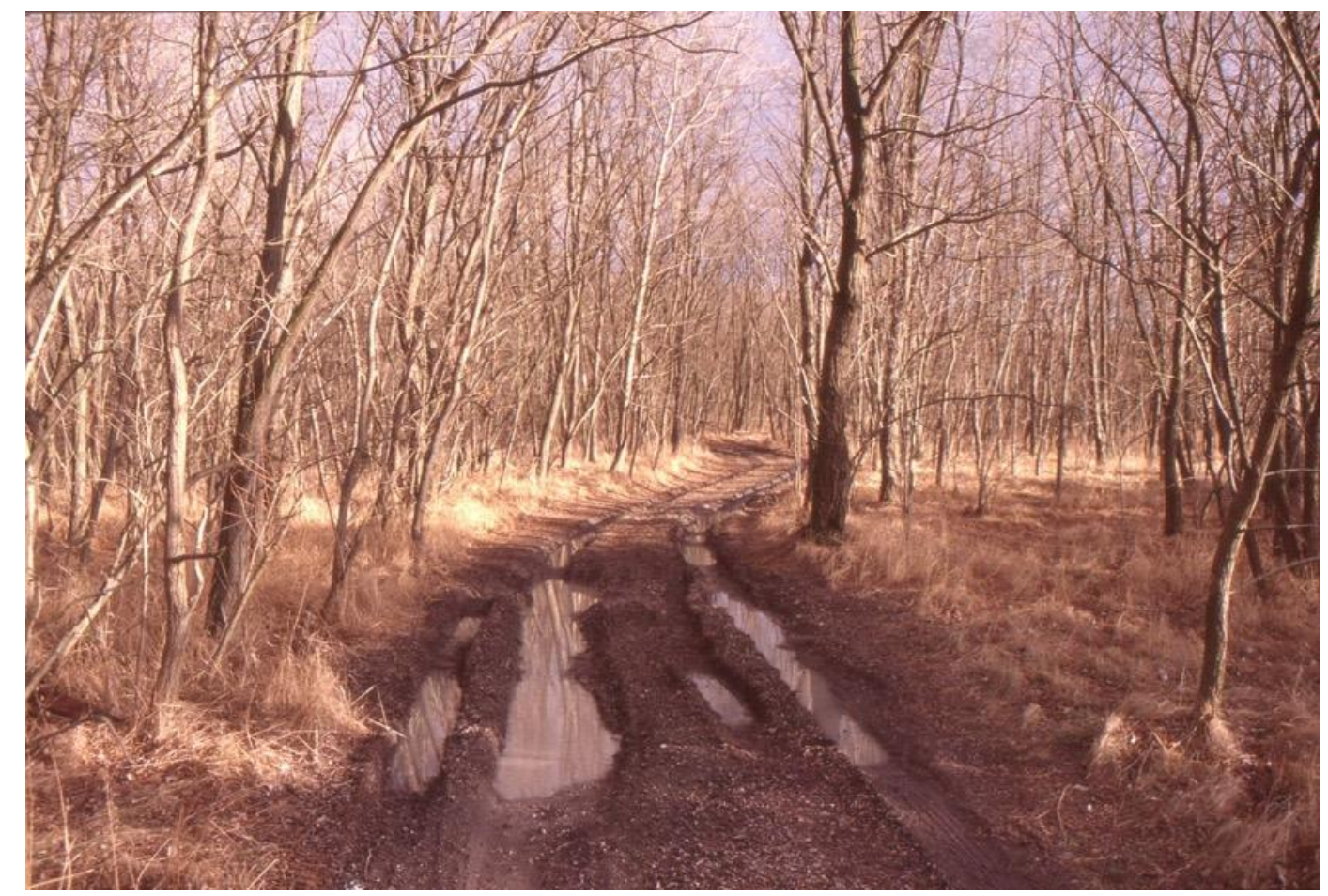

IIlustration 2 : Goitzsche-Wald, une forêt replantée sur la mine

\section{Un projet au chœur du Gartenreich}

La figure symbolique du Prince Franz von Anhalt-Dessau demeure aujourd'hui encore l'emblème d'une volonté de sagesse, d'éducation et de beauté dont les milliers de visiteurs se rendant à Wörlitz, Oranien-baum, Luisium et Sieglitzer, à quelques kilomètres de la Goitzsche, attestent de la vivacité. Il est donc normal que le projet de parc pour Bitterfeld s'inscrive dans cette tradition, sans bien entendu tenter en quoi que ce soit de la copier. Il s'agit plutôt de trouver un esprit moderne, à l'orée $\mathrm{du}$ XXIe siècle, qui fasse vivre une fois encore la volonté d'offrir les savoirs et les joies esthétiques qui ont fait la réputation du Gartenreich. Le nouvel équipement projeté n'est pas seulement une intervention matérielle dans un paysage naturel et urbain, c'est aussi, et peut-être avant tout, une manière nouvelle de s'adresser à nos concitoyens. Trois éléments en déterminent la philosophie :

\section{La relation au passé}

Les grandes heures de l'industrialisme sont derrière nous. Leur héritage a pour nous aujourd'hui un double visage : des valeurs sociales du travail et les monuments d'architecture industrielle et d'urbanisme qui leur sont liés. Il contient cependant aussi l'aliénation des hommes, la destruction 
des paysages et la pollution des rivières et des sols. Comme tout héritage, celui de l'optimisme industriel est donc ambivalent. C'est pourquoi il ne faut pas le refouler mais le travailler dans une perspective qui sache intégrer les nouveaux paramètres de la modernité, tels que le développement durable, la protection de la nature et la formation de l'homme à la nouvelle citoyenneté exigée par les règles de la démocratie active.

\section{Une action qui respecte l'environnement}

Par environnement nous entendons aussi bien l'espace urbain dans lequel les hommes vivent au quotidien que l'ensemble des rapports que ceux-ci tissent avec la nature dans le cadre de leurs activités industrielles ou de loisir.

\section{Les plaisirs de la promenade}

Le temps libre, lorsqu'il n'est pas le résultat d'un manque de travail, est destiné aujourd'hui au repos mais aussi, et de plus en plus, il est vécu comme une occasion de découverte, parfois même de formation. Cette fonction formatrice est d'autant plus importante que chacun est désormais appelé dans sa vie professionnelle à changer plusieurs fois de métier ou de secteur d'activité. L'ouverture d'esprit que favorise la découverte de la nature et des interventions artistiques qui lui ont donné une forme dans le Goitzsche-Wald, constituera ainsi un enrichissement pour tous ceux qui se laisseront entraîner dans cette expérience inédite.

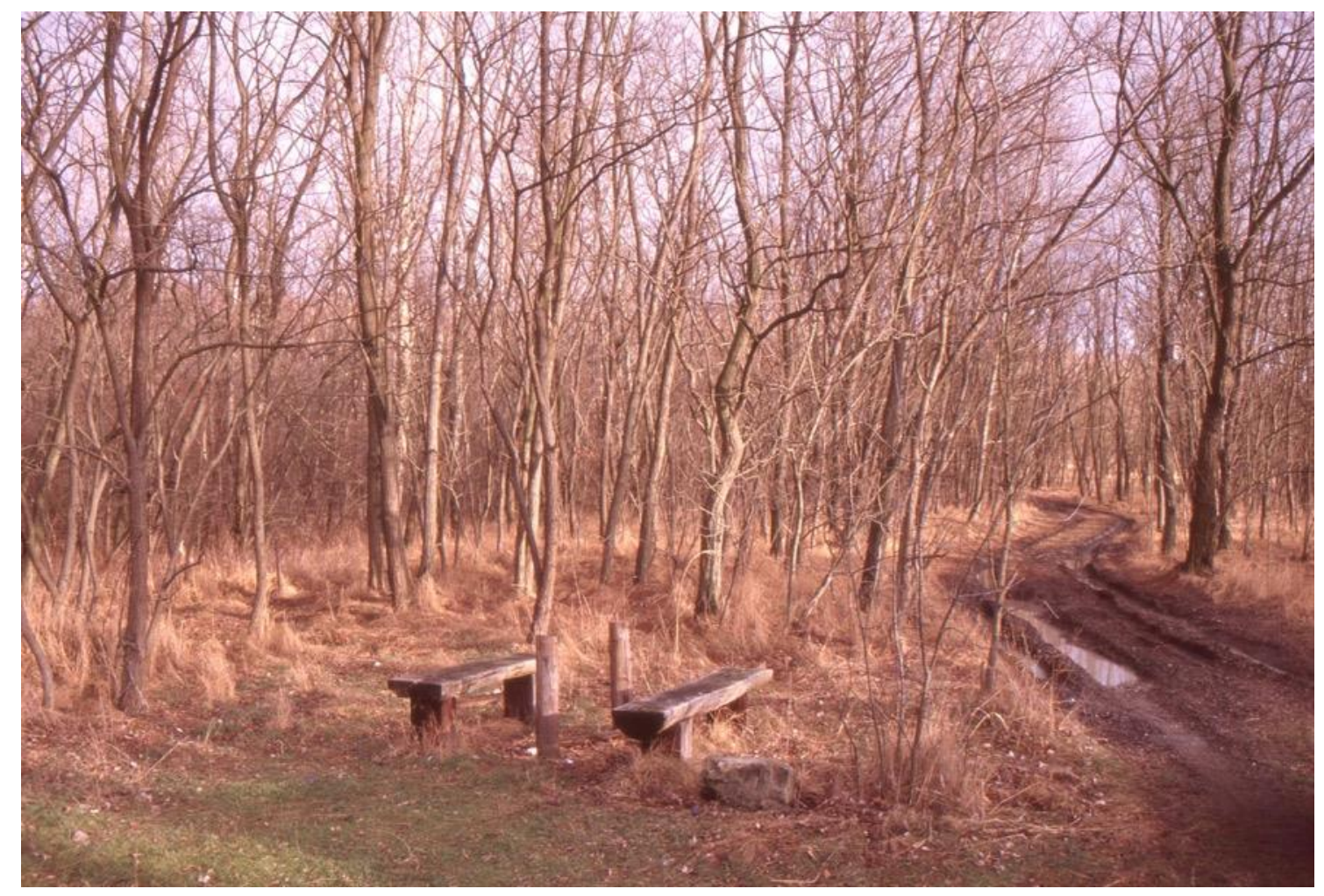

IIlustration 3 : redécouvrir les plaisirs de la promenade dans une forêt marquée par l'histoire

\section{Le Carré vert}

Juste au sud de la ville de Bitterfeld, la forêt s'étend presque jusqu'aux dernières maisons de la ville et prolonge en quelque sorte le «poumon vert » formé par les deux parcs urbains. C'est un très vaste espace résultant d'une replantation effectuée dans les années soixante sur l'emplacement du premier carreau de la mine. Il s'agit là d'un exemple de replantation typique de cette période qui, par ses caractéristiques formelles, constitue une transition entre le caractère urbain des faubourgs et 
le paysage qui se développe au-delà. Ce qui frappe d'abord, c'est la nature même de cette forêt, son artificialité. En cela, elle se distingue fortement des autres espaces de la Goitzsche comme de tout type habituel de forêt. Les arbres qui la constituent ont, après quarante ans, une belle présence, ce qui ne suffit pas à faire que les promeneurs s'y rendent par plaisir et la considèrent comme autre chose qu'un passage menant vers d'autres lieux.

Le Goitzsche-Wald est irrémédiablement perçu comme une forêt laide et inhospitalière, sauf par les quelques chasseurs qui y traquent le sanglier. Cette appréciation négative tient beaucoup à ce que, du fait de ses structures géométriques très apparentes, elle ne ressemble guère à ce qu'il est convenu d'appeler une «vraie, belle et bonne » forêt. Les traces qui témoignent de l'exploitation minière encore récente contribuent aussi à cette vision dépréciative. Le mythe romantique de la forêt a peine à y retrouver ses images favorites. Le Goitzsche-Wald est un territoire totalement artificiel issu de l'activité minière. Des structures de replantation mono-spécifiques, une topographie nonnaturelle faite de remblais, de nombreux témoignages de l'activité industrielle, tels que des lignes de chemin de fer, des pylônes électriques, des signaux et des voies de transport faites de plaques de béton. Dans ce paysage, les collines ne sont pas des collines, les lacs ne sont pas des lacs, les îles pas des îles; tout n'est que remblais, excavations et haldes. La forêt est par ailleurs traversée par un calepinage de chemins se coupant à angle droit, de profondes trouées et une route médiane asphaltée qui enserre partiellement cet espace dans sa moitié ouest. Dans cet univers rectiligne, un unique chemin curviligne traverse d'ouest en est : nous l'avons nommé le croissant de lune. 


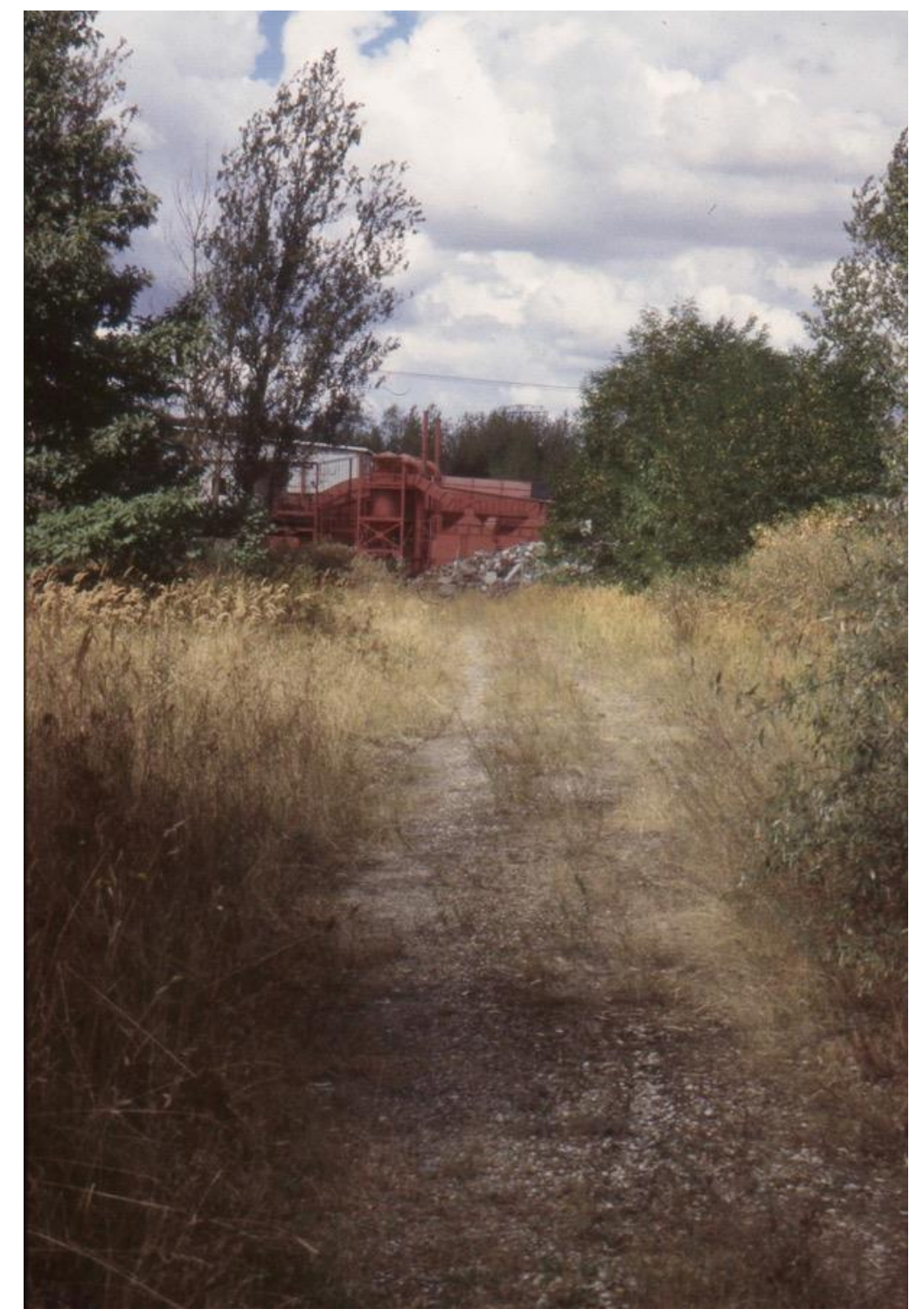

Illustration 4 : les témoignages de l'ancienne activité industrielle sont omniprésents

\section{La Forêt de Goitzsche : un livre ouvert sur la remise en culture des espaces miniers}

Les fortes structures qui caractérisent la Goitzsche renvoient à une histoire aujourd'hui dépassée. Elles sont cependant l'occasion, avec leurs contrastes violents, d'un sentiment du sublime, elles témoignent d'une «beauté du diable » illustrant exemplairement la relation ambivalente que notre société technique entretient avec la nature. Comme dans l'architecture industrielle qui l'a accompagnée, on peut y lire un signe du sentiment de toute puissance du progrès technique digne d'être médité et qui recèle également une beauté singulière. Cette forêt constitue un document unique sur la foi dans la toute puissance de l'homme. La replantation a été effectuée selon un schéma géométrique clair : essentiellement des surfaces carrées ou rectangulaires, à l'origine monospécifiques, plantées de peupliers (Populus nigra), pins sylvestres (Pinus silvestris), chênes pédonculés (Quercus pedonculata) ou chênes rouges (Quercus rubra), bouleaux (Betula alba), tilleuls (Tilia grandifolia) et acacias (Robinia pseudacacia), ces derniers dominant. 
Cette forêt très dense est entourée de quelques champs et d'espaces ouverts où s'est produit un phénomène classique de reconquête par des pionnières: spirée de Douglas (Spiraea douglasii), genets, églantiers (Rosa canina), oliviers de Bohême (Eleagnus angustifolia), baguenaudiers (Culetea arborescens), ou des essences déjà installées dans la région.

Là-dessus s'est surimposé un trait propre à l'économie réelle de l'époque de la République démocratique allemande (DDR) sous forme de ces hasards de livraison qui faisaient qu'on plantait ce qui arrivait dans les camions, «comme ça venait », racontent aujourd'hui encore les gens de la mine. C'est ce qui a produit cette configuration surprenante, qui résulte d'un processus de replantation à la fois rigide et aléatoire dans ses formes. On peut donc dire que, du point de vue écologique et esthétique, cette forêt témoigne des règles et des pratiques de replantation des années soixante, et c'est à partir de cet état que le concept d'aménagement de ce paysage a été élaboré.

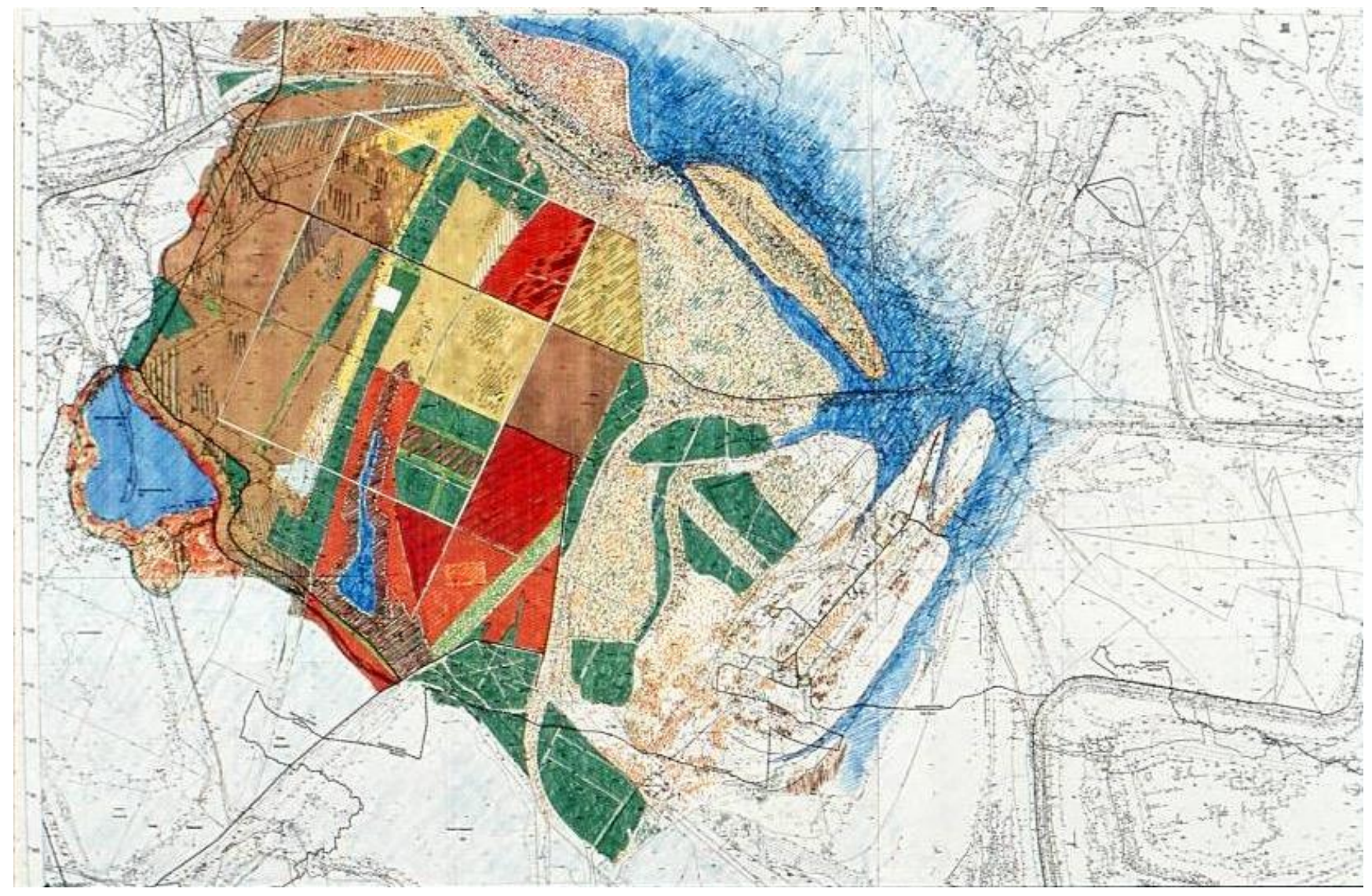

Illustration 4 : Cartographie des plantations : chaque couleur représente une espèce d'arbre différente (couleur unie : plantation initiale ; hachures : peuplement spontané).

\section{Des principes esthétiques pour l'entretien de la forêt}

L'idée directrice d'aménagement de la forêt de Goitzsche repose sur le respect de son artificialité et de la beauté qu'elle doit à cette intervention omniprésente de l'homme. Nous voulons donc la traiter comme une œuvre d'art, comme une «peinture » ou une «sculpture » des années cinquante. Cela passe d'abord par un travail sur les structures techniques qui marquent cet espace, sur leur renforcement, leur radicalisation, afin de donner une dimension esthétique à la perception qu'on en a. Pour les mêmes raisons on y introduira au contraire des ruptures. Dans son ensemble, le projet vise à réemployer $95 \%$ de l'existant grâce à cette stratégie novatrice. Par des ajouts et des retraits, par des formes diverses d'amélioration, ou simplement en laissant la nature faire seule son travail, nous voulons donner une force nouvelle à cet héritage écologique et formel. Un tel concept se distingue radicalement des stratégies d'aménagement habituelles qui consistent en Allemagne à 
choisir entre «exploitation forestière », «forêt écologique de loisir » ou «zone naturelle protégée ». Ici, les structures de l'occupation de l'espace, les perspectives ouvertes et le réseau de circulation seront traités esthétiquement dans le plein respect des principes écologiques de forestage. Autrement dit, les soins écologiques à apporter seront conçus dans l'esprit d'une élaboration esthétique du lieu.

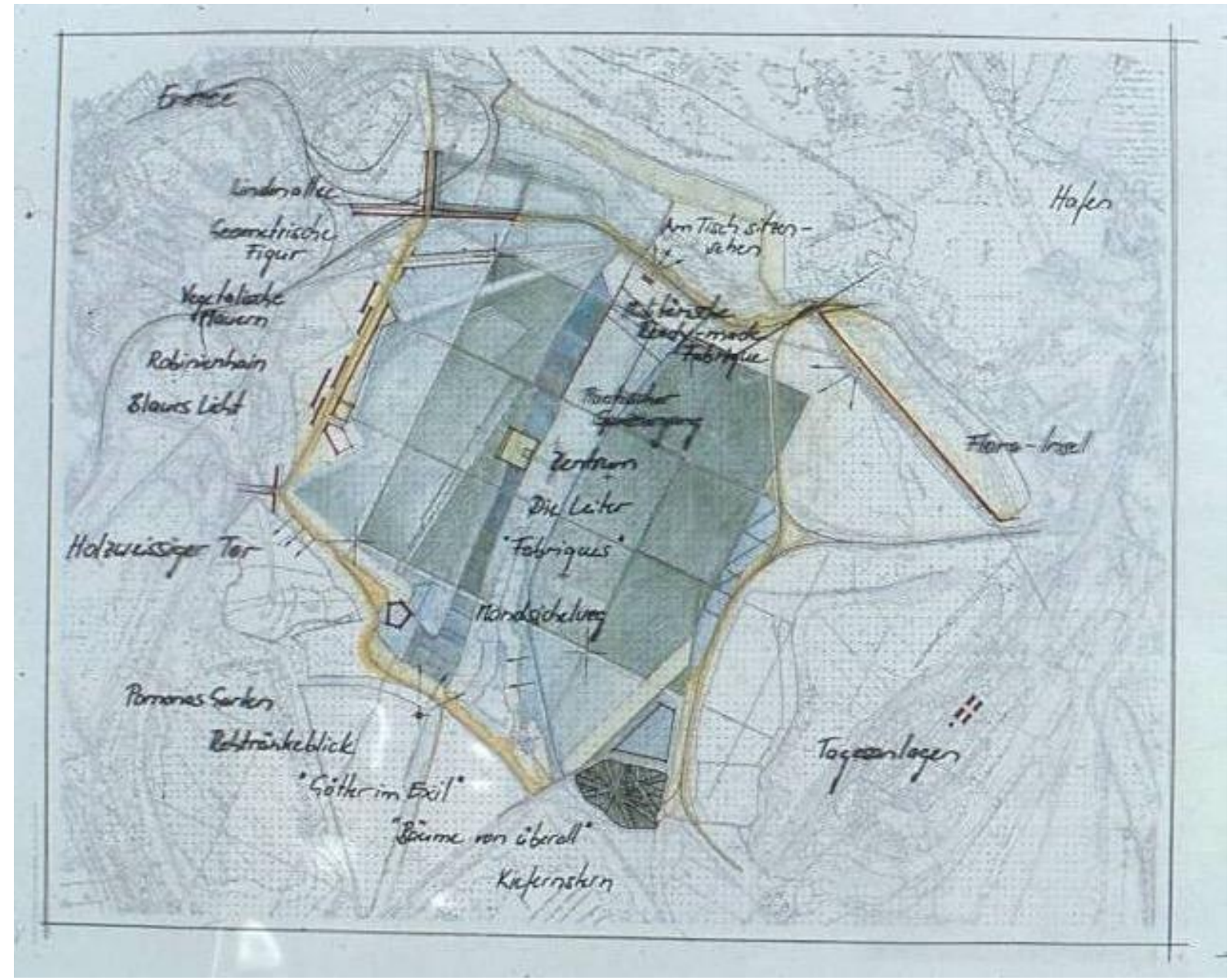

Illustration 5 : Plan de conception du Carré Vert de Goitzsche Wald (document de travail) 
Transformer un espace technique en parc exige qu'on apporte un soin particulier à la manière dont cet espace sera vécu par ses visiteurs. En particulier on visera à multiplier les expériences sensibles qui y seront possibles. Il faut attirer le visiteur et, sans vouloir le guider de manière contraignante, l'inviter à pénétrer dans cet espace inconnu et pour lui encore marqué par un passé peu attrayant. La stratégie consiste à lui offrir des surprises esthétiques, à lui donner l'impression d'une découverte permanente de trésors inattendus. Il ne s'agit pas d'un " programme » au sens que ce terme pouvait avoir dans la conception du Parc de Wörlitz, mais bien d'une intention, d'une guidance, qui doit être perceptible tout en préservant la liberté de mouvement du visiteur. Il faut donner l'impression qu'il y a mille choses, lieux, objets, sensations à découvrir, et ne pas décevoir cette attente.

Dans cet esprit plusieurs stratégies sont mises en œuvre. Dès l'entrée, l'aménagement d'une rampe par laquelle il pénètre donne au visiteur, qui aura abandonné sa voiture au parking ou sera arrivé à pied, l'impression qu'il entre dans un espace distinct: il le découvre d'une hauteur qui permet de saisir cette forêt comme un objet à part dans le grand paysage. Dès l'abord, le visiteur en percevra aussi les grandes structures et saura qu'il se trouve dans un espace différent des forêts qu'il connaît alentour. Du haut de ce petit promontoire, et pour l'inviter à aiguiser son regard, il pourra « encadrer » des fragments de paysages dans des cadres métalliques installés là à son intention, qui l'aideront à fragmenter sa perception, à l'organiser, à construire à son gré différents cadrages organisant des objets-paysages partiels.

Après cette phase ludique, qui lui aura par ailleurs permis de distinguer les chemins qui s'offrent à lui à quelques dizaines de mètres, il pourra s'engager dans l'une ou l'autre des directions offertes. Au fur et à mesure de sa progression, des chemins singularisés par des espèces végétales spontanées (lupins, roses sauvages, genêts, etc.) alterneront avec des objets, que nous désignons du terme traditionnel de «fabriques », qui créeront pour lui un rythme dynamique de promenade. Ces «fabriques » sont, suivant les cas, des objets préexistants laissés par l'activité industrielle ou des mini-monuments créés aux fins de susciter l'intérêt esthétique et la surprise. Ils ont à la fois une valeur émotionnelle, comme tout objet d'art, et une fonction structurante dans le parcours général de l'espace. Les "fabriques », malgré leur petit nombre dans l'immensité de cette forêt, doivent renforcer le sentiment de se promener dans un parc, d'y être invité de façon ludique et sensible. Ces «événements » doivent aider à faire de la promenade une véritable découverte du paysage et des singularités de cette nature artificielle. 


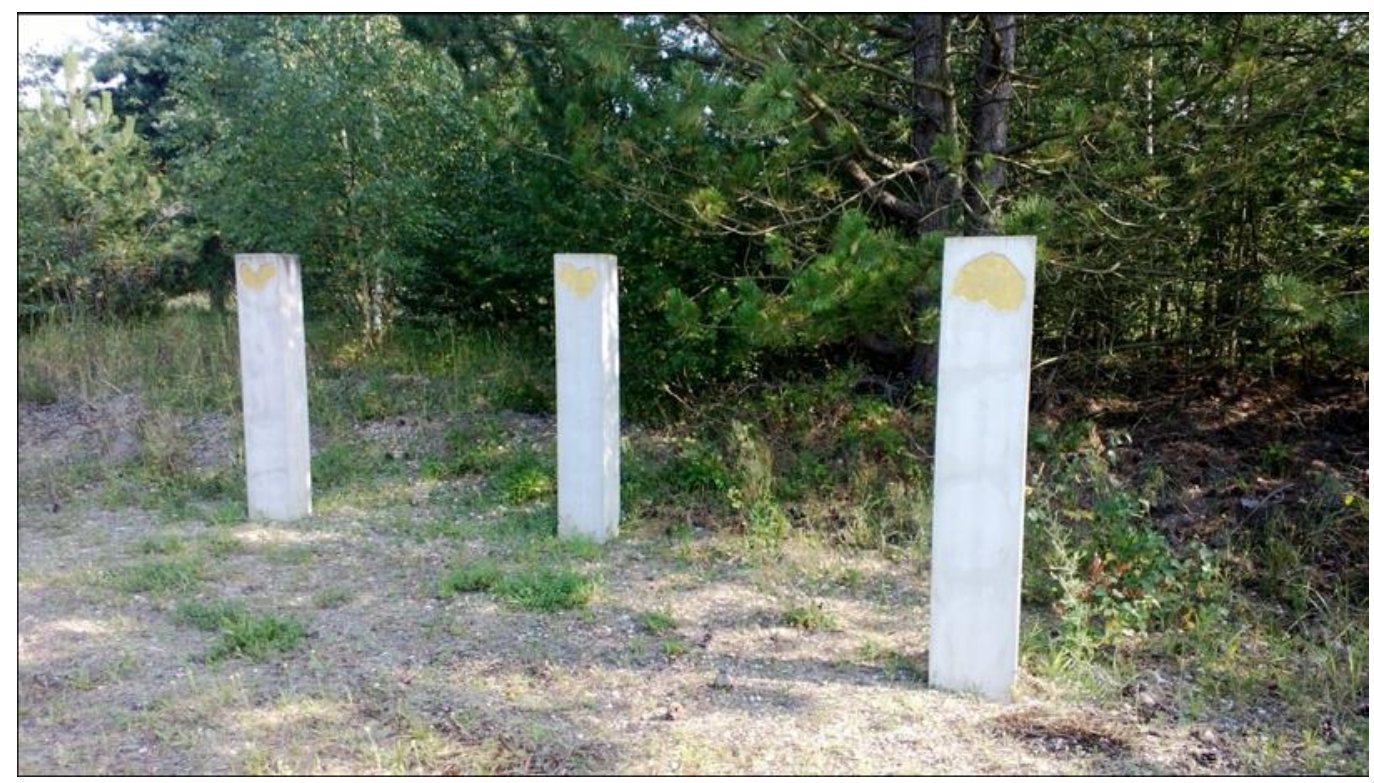

Illustration 6 : une des "Fabriques" à découvrir dans la forêt, le sentier des papillons.

Dans cet esprit, les nombreuses perspectives ouvertes dans le couvert pour les besoins de l'exploitation feront l'objet d'un soin particulier. On soulignera leur présence par des aplats de couleur en plantant à leur bord par exemple des spirées de Douglas. Les ruptures dans le continuum visuel prennent d'ailleurs souvent une forme très inattendue, comme par exemple la longue « échelle » qui divise le territoire, alignant quinze petits carrés de pins sylvestres de un hectare chacun. On profitera de cette particularité pour y installer, comme dans des « chambres », des «fabriques » plus travaillées comme une Conversation piece ou un labyrinthe.

\section{Un centre ouvert}

Non loin de là, se trouve le cœur géographique de la forêt, également situé dans les « chambres » carrées de pins sylvestres. Nous y ouvrirons une clairière d'environ deux hectares, au centre de laquelle sera construit le centre symbolique du territoire, le foyer du Goitzsche-Wald. Vaste structure excavée de trente mètres de côté et d'un mètre trente de profondeur, cernée de murs de brique, elle offrira la possibilité de s'asseoir, converser ou jouer de la musique. Image inversée du poumon vert dans la ville, le centre sera comme une mise en scène des rapports entre les éléments construits, la clairière et l'espace sauvage environnant. Il renforcera symboliquement l'idée d'un dialogue à plusieurs voix entre l'homme et la nature, dans un espace convivial. 


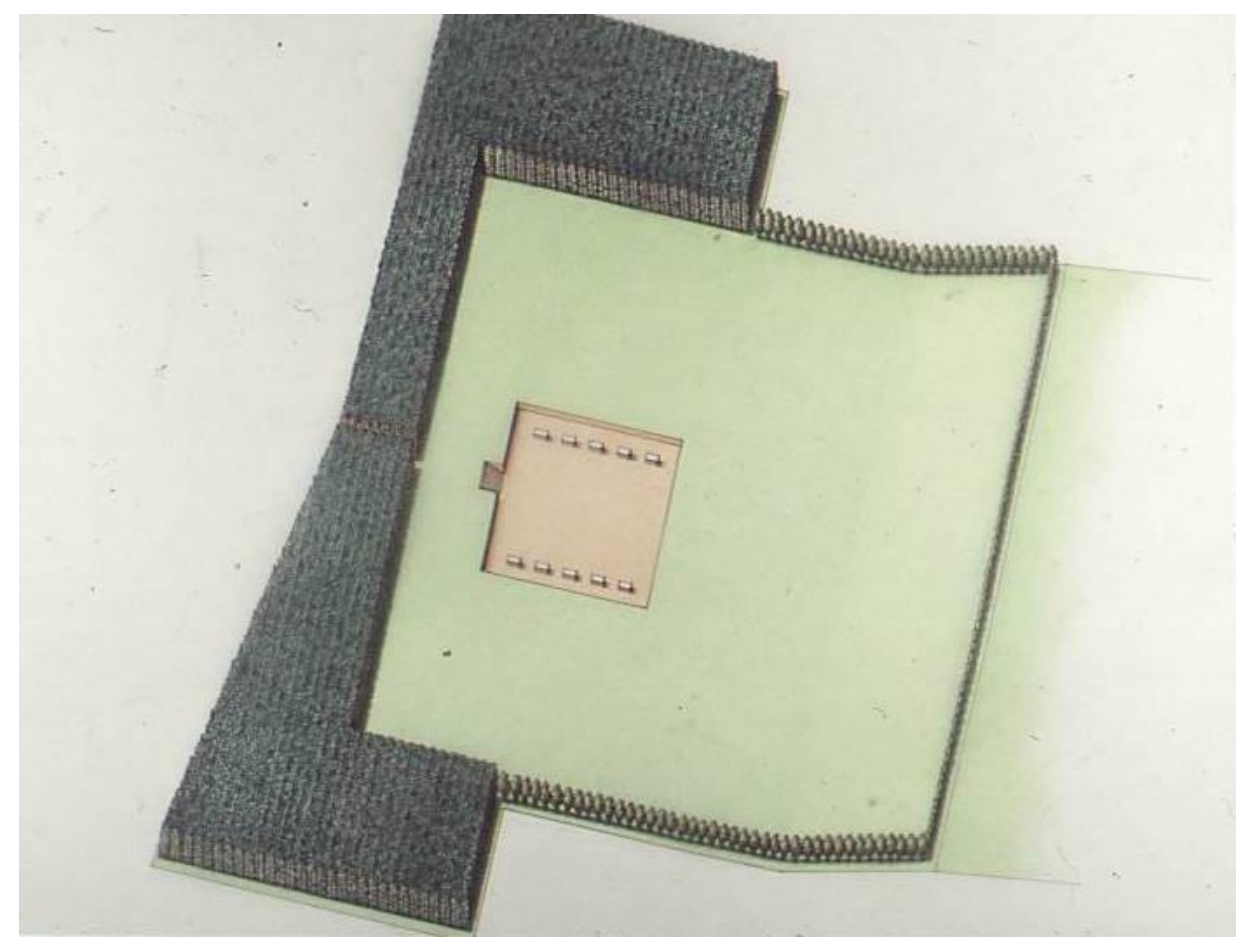

Illustration 7 : le Centre ouvert (dessin de conception)

Le carré est, en soi, une des formes essentielles dont notre culture a hérité. En art, Joseph Albers qui fut de 1925 à 1932 «Maître » au Bauhaus de Dessau, à quelques kilomètres, l'utilisa dans son enseignement sur l'acuité de la vision et lui consacra la célèbre série de ses «Hommages au carré » (Homage to the Square). Cette activation du regard, disait Albers, doit renforcer notre attention au monde environnant et par là conduire l'homme à un meilleur comportement à son égard. Par sa forme carrée, le centre figure donc la philosophie même du projet en offrant un point de rencontre pour les hommes au cœur de la nature, l'idée d'une réconciliation entre eux, motif encore fortement utopique à l'orée de ce XXIe siècle. Nous souhaitons que le carré, donnée héritée du processus de replantation, soit l'occasion pour le visiteur, au cœur de cette forêt, de se ressaisir comme Homme, comme être de culture au sein de la nature. Depuis toujours, notre tradition culturelle a éprouvé à l'égard de celle-ci des sentiments ambivalents, qui vont de l'amour au besoin de détruire pour se protéger et survivre. Au cours des siècles, cette ambivalence s'est exprimée dans la langue par des séries de notions opposées deux à deux qu'on retrouve dans les descriptions de l'expérience de la nature des philosophes et des écrivains :

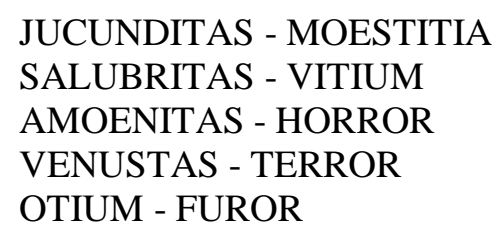

Sur les bancs qui l'accueilleront dans le centre, le promeneur retrouvera ces notions gravées dans la pierre, comme une invite poétique à réfléchir lui-même sur sa place dans la nature. 


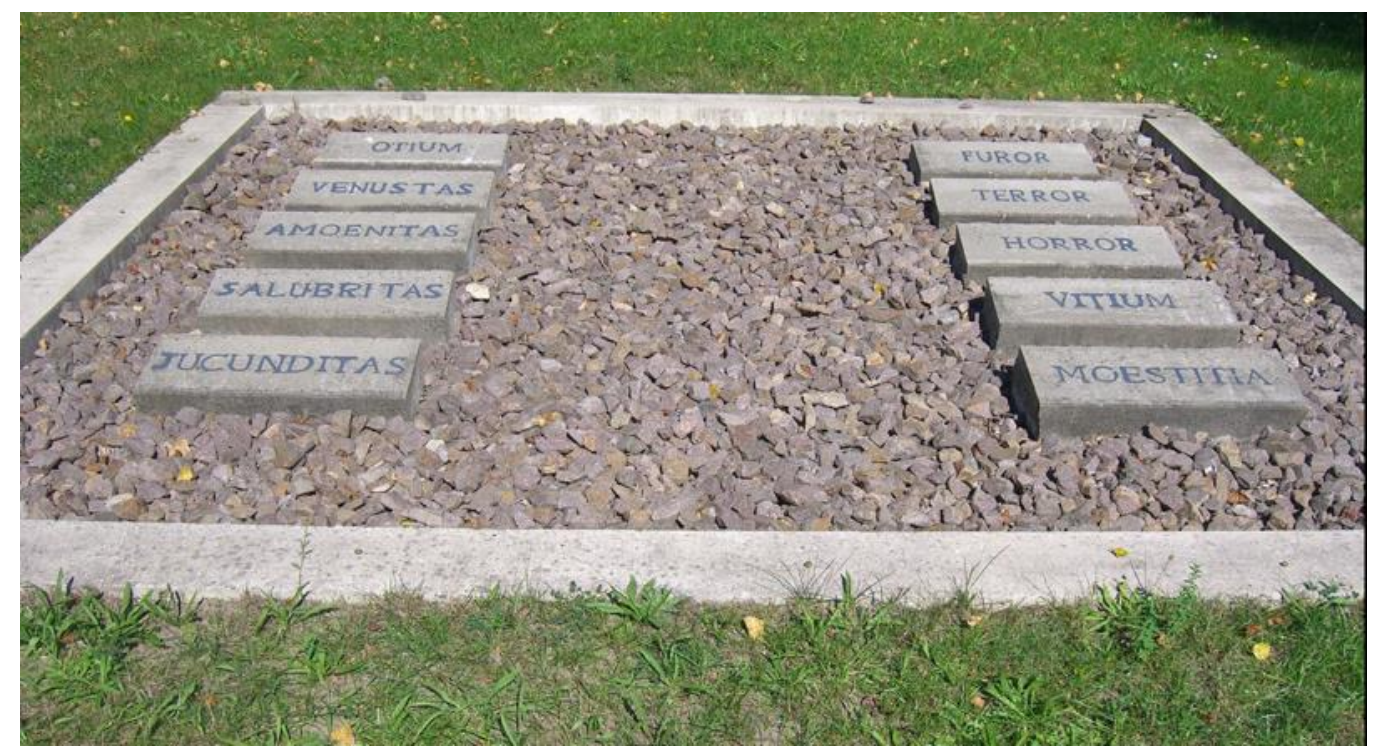

Illustration 8 : Une installation poétique évoquant l'ambivalence de nos relations à la nature

À ce jour, une grande partie de ce projet a été réalisée dans ses axes fondamentaux, avec des modifications formelles qui n'en ont pas émoussé les principes de base. 